ARTIGO

Recebido em: 28/04/2017

Aceito em: 09/04/2018

\title{
Memória organizacional em apoio às decisões em uma empresa do setor tecnológico
}

\author{
Organizational memory to support decisions in a tech sector \\ company
}

\author{
Jessica TAVARES (jehcriss42@gmail.com)* \\ Newton de CASTILHO JUNIOR (ncastilho@ufpr.br)* \\ * Universidade Federal do Paraná.
}

\begin{abstract}
Resumo
Aborda a memória organizacional, como processo da Inteligência Organizacional, de modo a identificar como essa pode otimizar a tomada de decisão. 0 enfoque informacional é o de recuperação da informação vinculada aos processos, instruções de trabalho, ferramentas e técnicas da organização. A pesquisa busca responder se a empresa possui memória organizacional, com foco na recuperação da informação, de forma a otimizar o processo de tomada de decisão. A pesquisa, caracterizada como qualitativa e descritiva, com suporte da literatura sobre o tema, foi aplicada a uma empresa do setor tecnológico. 0 resultado apontou que a empresa tem políticas que visam dar suporte à incorporação dos processos e a melhorias contínuas ao usar experiências que permitem incrementar o acervo de informações e conhecimentos da organização. Contudo, possuí deficiências quanto à recuperação da informação, impactando negativamente o processo de tomada de decisão. 0 estudo propõe atenção às deficiências na recuperação de ativos de informação registrados na memória, em um contexto de inteligência organizacional.
\end{abstract}

Palavras-chave: Memória Organizacional. Recuperação da Informação. Inteligência Organizacional. Suporte à Decisão.

\begin{abstract}
It addresses organizational memory, as an Organizational Intelligence process, in order to identify how it can optimize decision-making. The informational approach is the information retrieval linked to the processes, work instructions, and organization tools and techniques. The research seeks to answer if the company possesses organizational memory, focused on information retrieval, so that it can optimize the decision-making process. A qualitative and descriptive research, supported by the literature on the subject, was applied to a company from the technological sector. As results, we found that the company has policies that aim to support the incorporation of processes and continuous improvements by using experiences that allow increasing the collection of information and knowledge of the organization. However, it revealed deficiencies in the information retrieval tasks with negative impacts to the decision making process. The study proposed attention to the deficiencies in retrieving information assets from memory, in an Organizational Intelligence context.
\end{abstract}

Keywords: Organizational Memory. Information Retrieval. Organizational Intelligence. Decision Support.

v. 23 , n. esp., 2018 p. $50-59$

ISSN 1518-2924
(ㅇ) (1) Esta obra está licenciada sob uma Licença Creative Commons. 


\section{INTRODUÇÃo}

A tecnologia da informação e comunicação e a crescente produção de dados e informações têm impacto significativo na sociedade atual e, por consequência, nos estudos ligados à informação e conhecimento. Isto pode ser percebido na própria designação da sociedade contemporânea, tida como "sociedade da informação", em que a ideia subjacente concentra-se no processo de mudança constante, pelo avanço da ciência e tecnologia. (COUTINHO, 2011, p.6).

Outra expressão utilizada para designar a sociedade atual é "sociedade do conhecimento" que quanto ao discurso inclinado ao mercado, remete às suas exigências, velocidade de fluxos de capital e informação, avanços tecnológicos, alta produtividade em massa. (ABREU, 2009, p.1-2).

Neste cenário a organização deve saber gerenciar seus dados, informações e conhecimento de forma a se manter competitiva e sobreviver no mercado. A prática de gerenciamento da informação está contextualizada no ciclo informacional, de Ponjuán Dante (1998 apud Tarapanoff, 2006), no qual inclui-se elementos como a geração, seleção/aquisição, representação, armazenamento, recuperação, distribuição e uso da informação.

Um importante elemento a ser considerado neste contexto é a memória organizacional que, segundo Conklin (2001 apud Freire et al. 2012), amplia o conhecimento por capturar, organizar, divulgar o conhecimento criado pelos trabalhadores dentro de uma empresa.

Por sua vez, a Inteligência Organizacional é definida como a "capacidade de uma organização para processar, interpretar, codificar, manipular e acessar informações de maneira proposital e direcionado a metas, para que a organização possa aumentar seu potencial de adaptação no ambiente que opera" (GLYNN, 1996, p.1088, tradução dos autores).

O problema identificado nesta pesquisa é a falta de incorporação dos procedimentos e instruções de trabalho no dia a dia, que acarreta na falta de participação dos colaboradores para a continua melhoria dos processos. Existem procedimentos, instruções de trabalho, ferramentas, técnicas e regras que são documentados, e há o esforço em gerar informação que contribua para a melhoria contínua, como lições aprendidas no fim de cada projeto. Porém, essas ficam em repositórios pouco acessados e as informações são pouco disseminadas, limitando assim a possibilidade da organização em reutilizar o conhecimento da experiência.

Compreende-se que a incorporações dos processos e possibilidades de melhorias, ao incrementar as experiências no processo, contribui para que as melhores decisões sejam tomadas com base no repositório de informação e conhecimento da organização.

A questão que este estudo busca responder é: a empresa possui memória organizacional, com foco na recuperação da informação e do conhecimento, de forma que possa otimizar o processo de tomada de decisão?

Desta forma, o objetivo deste estudo é avaliar os aspectos de recuperação de informação e conhecimento a partir da memória organizacional, visando à otimização do processo de tomada de decisão no contexto da Inteligência Organizacional.

\section{PROCEDIMENTOS METODOLÓGICOS}

Esta pesquisa é de caráter descritiva que, segundo Gil (2008), tem como objetivo primordial a descrição das características de determinado fenômeno e qualitativa, uma vez que, conforme aponta Flick (2009), utiliza o texto como material de estudo e está 
interessada na perspectiva dos participantes, em suas práticas do dia a dia e em seus conhecimentos.

A fonte utilizada para diagnóstico da organização é o resultado de uma avaliação externa da organização, chamada The Standard CMMI Appraisal Method for Process Improvement (SCAMPI) do Capability Maturity Model - Integration (CMMI), no qual a empresa estudada é certificada e está em processo de recertificação.

Existem três classes de avaliação: SCAMPI A, B e C. O SCAMPI A é o oficialmente reconhecido e o método mais rigoroso ${ }^{1}$. Por sua vez, a classe $\mathrm{B}$ e $\mathrm{C}$ são menos formais $\mathrm{e}$ contribuem para ajudar a organização a identificar oportunidades de melhorias. (TEAM, SEI CMMI Production, 2010).

Para esta pesquisa foi utilizado o resultado do SCAMPI B para identificação do problema de Inteligência Organizacional por ser a avaliação mais recente finalizada e disponível na organização e também os dados dos repositórios de informação da empresa.

O SCAMPI B ocorreu durante cinco dias, nos quais um time composto por integrantes da empresa e um consultor externo realizaram entrevistas e avaliação dos produtos de trabalho, revisando os processos com apoio de afirmativas e evidências. Os entrevistados foram os diretores da organização, responsáveis pela gestão das áreas e colaboradores que participam de alguma fase do processo. A relação de pontos fracos e recomendações consideradas pertinentes a este estudo estão detalhados na sessão 4 Resultados: apresentação e discussão.

\section{DESENVOLVIMENTO CONCEITUAL}

As bases teóricas principais utilizadas para o desenvolvimento deste estudo foram: o modelo inteligência organizacional/ciclo de aprendizado apresentado por Choo (2011) e os imperativos da estrutura da memória organizacional, apresentados por Walsh e Ungson (1991). Desta forma, é proposto uma avaliação da recuperação da informação, de forma a analisar a influência da memória para a tomada de decisão.

\subsection{Tomada de decisão nas organizações}

Conforme apontam Prevé, Moritz e Pereira (2010), os processos de tomada de decisão são constantes no dia a dia da organização e a todo o momento as pessoas são colocadas em uma situação em que é necessário analisar, investigar, optar e agir frente às poucas ou muitas opções que lhes são fornecidas. Estes autores salientam ainda que existe o desafio dos tomadores de decisão em pensar globalmente e de usar instrumentos de informação e de comunicação que venham a colaborar no processo decisório. É ressaltado que:

Um processo de tomada decisão inicia-se pela identificação das necessidades, no que é possível fazer, da informação que está disponível e da comunicação que precisa ser efetuada. Esperamos que esses elementos, ordenados em uma estrutura lógica, resultem na possibilidade de uma melhor decisão. (PREVÉ, MORITZ e PEREIRA, 2010, p.77).

Maximiano (2000) menciona que a forma como os gerentes tomam decisões para resolver problemas é influenciada por alguns fatores inerentes ao próprio

1 O SCAMPI A incorpora as melhores práticas reconhecidas como bem sucedidas na comunidade de avaliação. A avaliação é coordenada por avaliadores certificados pelo Software Engineering Institute (SEI). (TEAM, SEI CMMI Production, 2010). 
problema, que são criados pelo ambiente ou fazem parte das competências e informações do tomador de decisões. A recuperação da informação é importante neste contexto, uma vez que dada a necessidade de obter informações cada vez mais rápidas, surgem novas tendências que visam recuperar a informação de forma acelerada (PREVÉ, MORITZ e PEREIRA, 2010). Tarapanoff (2006,) indica que, além da informação e o conhecimento, a inteligência também está presente nos processos de gestão da organização, assim como alimentam o processo de tomada de decisão e o planejamento estratégico. Desta forma, está diretamente ligada à inteligência organizacional.

\subsection{Inteligência organizacional}

Müller e Castilho Júnior (2012) apontam que a Inteligência Organizacional atua no ambiente externo e interno da organização, no qual o primeiro é acompanhado com auxílio de atividades de coleta de dados e informações sobre itens como o setor, concorrentes, clientes, fornecedores assim como o contexto político-legal, tecnológico, demográfico, cultural, entre outros, que a empresa está inserida; o segundo pode ser trabalhado por meio de atividades que consigam captar informações e conhecimentos, armazená-los e comunicá-los aos membros e utilizar as informações e conhecimentos para os processos de tomada de decisão. Considerando-se aspectos informacionais da aprendizagem, o processo de inteligência/aprendizagem organizacional pode ser visto como:

[...] um ciclo contínuo de atividades que envolvem: sentir o ambiente, desenvolver percepções e gerar sentido por meio da interpretação, utilizando-se da memória sobre a experiência do passado para auxiliar a percepção e a execução da ação, com base nas interpretações desenvolvidas. Choo (2011, p.43).

Choo (2011, p.44) discorre sobre os elementos do processo da organização inteligente, esquematizado na Figura I.

Memória

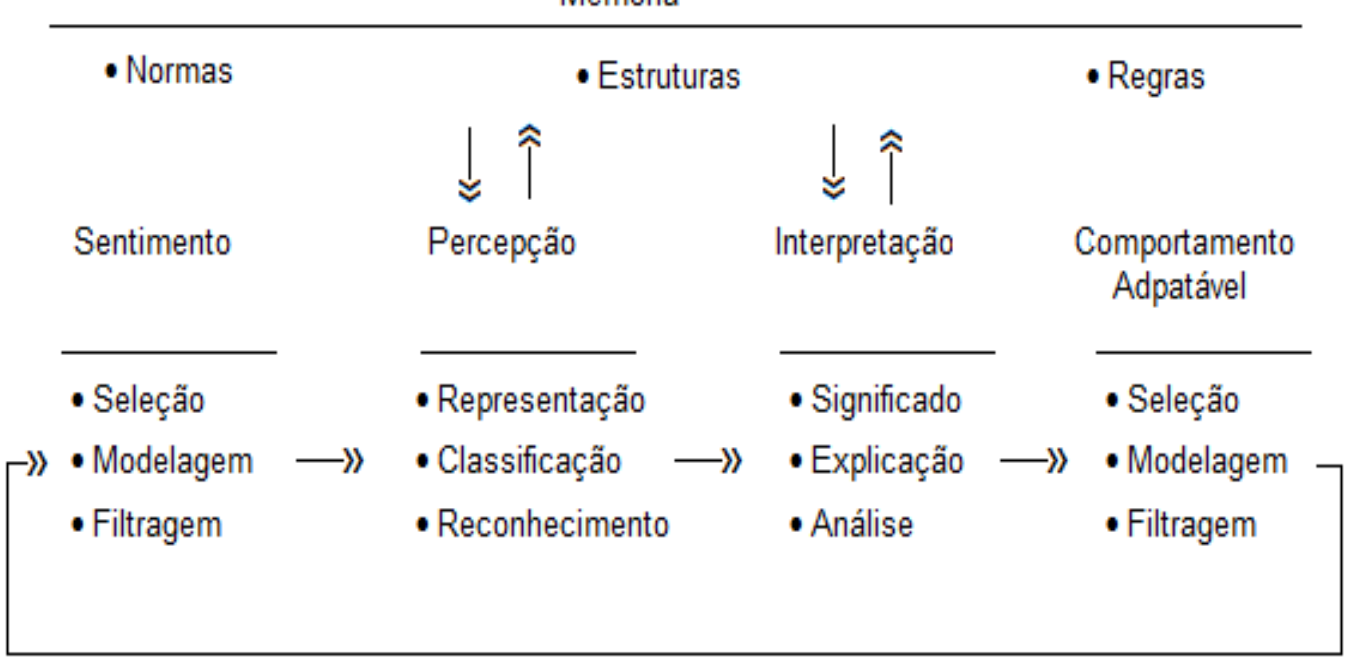

Figura 1: Inteligência organizacional/ciclo de aprendizado Fonte: Choo (2011).

No qual: a) o "sentimento" se refere a coletar as informações sobre os ambientes externos e internos, filtrar de acordo com seus interesses e selecionar áreas para uma análise mais apurada; b) a "memória" é derivada das experiências da organização; c) a "percepção" refere-se à identificação de eventos e entidades externas e desenvolvimento de 
descrições sobre eles utilizando o conhecimento descrito na memória; d) a "interpretação" está no centro do ciclo da inteligência, de modo a tentar explicar o que está acontecendo de modo significativo para a organização; e) o "comportamento adaptativo" refere-se ao inicio de um novo ciclo de aprendizagem na medida em que a organização toma decisões e realiza ações que geram efeitos e resultados. Pode-se perceber que a memória permeia todo o ciclo do processo de inteligência/aprendizagem.

\subsection{Memória Organizacional}

Molina e Valentim (2011) ressaltam que a memória vai além do que está representado pela mente humana, o corpo, o aparelho sensitivo e motor, o tempo físico, e inclui representações, rituais, textos e comemorações. Isto explica o interesse do estudo da memória em várias áreas do conhecimento científico, tais como psicologia, neurociência e história. Sendo que ao nosso estudo, interessam principalmente aquelas ligadas às técnicas de manipulação da informação (CRIPPA, 2010 apud MOLINA E VALENTIM, 2011).

A Memória Organizacional é ainda definida como:

acervo de informação, conhecimentos e práticas, agregados e retidos pela organização ao longo de sua existência, utilizados para o suporte às suas atividades, seus processos decisórios e para a preservação do seu capital intelectual, potencializando a gestão do conhecimento (MENEZES, 2006, p.31).

Walsh e Ungson (1991) basearam o estudo da memória organizacional em três premissas: a) que organizações assemelham-se a sistemas de processamento de informação, no qual a memória na organização é similar, em função, à memória individual; b) que além de sistema de processamento de informação, as organizações são representadas como sistema interpretativo; c) que organizações possuem uma rede de significados compartilhados que é sustentado pela linguagem comum e interações sociais. Os autores mencionam que a memória desempenha três papéis importantes na organização: o papel informacional - no qual o conteúdo de informação pode contribuir para uma eficiente e efetiva tomada de decisão; o papel de controle - pode servir para moldar o comportamento desejável sem incorrer em custos de monitoração; e o papel político - o controle de informação cria uma fonte de dependência com o qual indivíduos ou grupos com poder estão capacitados para influenciar as ações dos outros.

Walsh e Ungson (1991) ainda apontam que existem três imperativos para consideração da Memória Organizacional, a saber: a aquisição da informação - que consiste em conhecer os processos nos quais a informação é adquirida, armazenada e recuperada; a retenção da informação - que especifica a estrutura de retenção; e a recuperação da informação - que identifica os caminhos através dos quais o uso da memória influenciará nos resultados e no desempenho da organização, para então recuperá-la.

Telles, Karawejczyk e Borges (2014) afirmam que a recuperação da memória deve levar em conta: a) aprender com a experiência passada e acumulada - a memória acumulada auxilia na busca da experimentação e da inovação, reduzindo custos em decorrência da presença de procedimentos operacionais que podem ser consultados; b) aprender com os projetos postos em prática - através da memória organizacional é possível direcionar caminhos e decisões nos projetos.

\section{RESULTADOS: APRESENTAÇÃO E DISCUSSÃO}

A empresa estudada opera como desenvolvedora de sistemas e fornecedora de serviços de manutenção de sistemas. A empresa é certificada na norma ISO 9001/2008 e 
no modelo CMMI-dev com o nível 3 de maturidade, modelo que fornece o diagnóstico da empresa.

Como fonte do diagnóstico e detalhamento do problema foi utilizado o relatório gerado como resultado da avaliação externa denominado The Standard CMMI Appraisal Method for Process Improvement (SCAMPI) e as informações que constam na base de processo da organização.

Por meio destes, foi possível identificar algumas fraquezas e recomendações que podem ser avaliadas pela perspectiva da Inteligência Organizacional, conforme apresentado no QUADRO I e II.

Os pontos fracos destacados relativos à recuperação de informação remetem: ao armazenamento de documentos em locais incorretos, dificuldade em encontrar parâmetros utilizados em cálculos nos indicadores usados na organização, estrutura de arquivos e diretórios que não são feitos conforme definido nos procedimentos e instruções de trabalho, falta de identificação das ferramentas utilizadas para gestão de bugs dos projetos de desenvolvimento e ferramentas gerais utilizadas em projetos e, por fim, a dificuldade de identificar treinamentos organizacionais e de projeto, para melhor avaliação de custos, despesas, etc.

Quadro 1: Apresentação dos resultados Scampi B - fraquezas

\begin{tabular}{|c|l|}
\hline \multicolumn{2}{|c|}{ Fraquezas } \\
\hline 1 & Documentos não estão sendo colocados no local correto \\
\hline 2 & $\begin{array}{l}\text { Na definição dos indicadores é citado parâmetros de } \\
\text { cálculos, entretanto não encontramos a definição deste } \\
\text { termo. }\end{array}$ \\
\hline 3 & $\begin{array}{l}\text { Alguns projetos não seguem o padrão de estrutura de } \\
\text { arquivos e diretórios da organização, mantendo os } \\
\text { artefatos de referentes à interface em um diretório } \\
\text { específico quando deveriam fazer parte do artefato de } \\
\text { projeto/arquitetura. }\end{array}$ \\
\hline 4 & $\begin{array}{l}\text { Para garantir que os dados foram analisados também } \\
\text { seria necessário ter acesso à ferramenta que está sendo } \\
\text { usada para manter os bugs, porém não foi encontrada. }\end{array}$ \\
\hline 5 & $\begin{array}{l}\text { Para garantir que os dados foram analisados também } \\
\text { seria necessário ter acesso à ferramenta que está sendo } \\
\text { usada para manter os bugs, porém não foi encontrada. }\end{array}$ \\
\hline 6 & $\begin{array}{l}\text { Não foi identificado em todos os projetos todas as } \\
\text { ferramentas utilizadas em sua execução. }\end{array}$ \\
\hline 7 & $\begin{array}{l}\text { Na planilha de treinamentos não conseguimos identificar } \\
\text { quais treinamentos são organizacionais, ou específicos } \\
\text { do projeto ou grupo. Somente olhando solicitação por } \\
\text { solicitação de treinamento. }\end{array}$ \\
\hline
\end{tabular}

Fonte: elaborado pela autora (2016).

Quanto às recomendações, sugere-se a necessidade de se disseminar a existência das políticas organizacionais e a consolidação das informações em um número menor de locais. 
Quadro 2: Apresentação dos resultados Scampi B - recomendações

\begin{tabular}{|l|l|}
\hline \multicolumn{2}{|c|}{ Recomendações } \\
\hline 1 & Disseminar a existência das políticas organizacionais \\
\hline 2 & $\begin{array}{l}\text { Consolidar as informações em um número menor de } \\
\text { locais }\end{array}$ \\
\hline
\end{tabular}

Fonte: elaborado pela autora (2016).

A política organizacional foi destacada devido a sua importância quanto às orientações ou regras gerais da gestão da organização e sua relação com a inteligência da organização, uma vez que:

Do ponto de vista estratégico, as políticas organizacionais podem contribuir significativamente para a inteligência da organização. No que tange ao cotidiano da organização, as políticas permitem resoluções de forma padrão. E ainda podem uniformizar e facilitar a elaboração de atividades operacionais e processos de trabalho, reduzir o tempo para a tomada de decisões, melhorar a comunicação interna, mediar pressões, harmonizar comportamentos, minimizar atritos, combater desperdícios, evitar erros e economizar gastos (REZENDE, 2012 apud BASTOS, 2014).

Ao analisar a empresa, pode-se perceber que os processos, que se desenvolvem em procedimentos, instruções de trabalho, ferramentas, técnicas e regras são documentados e são orientados pela política organizacional.

Na Figura II está ilustrado como é a estrutura dos processos da empresa. Existe uma base de dados geral, onde constam todos os processos da organização, no qual o documento principal é o Manual da Qualidade, onde todos os procedimentos e instruções de trabalho são agregados, e descreve a definição da politica organizacional.

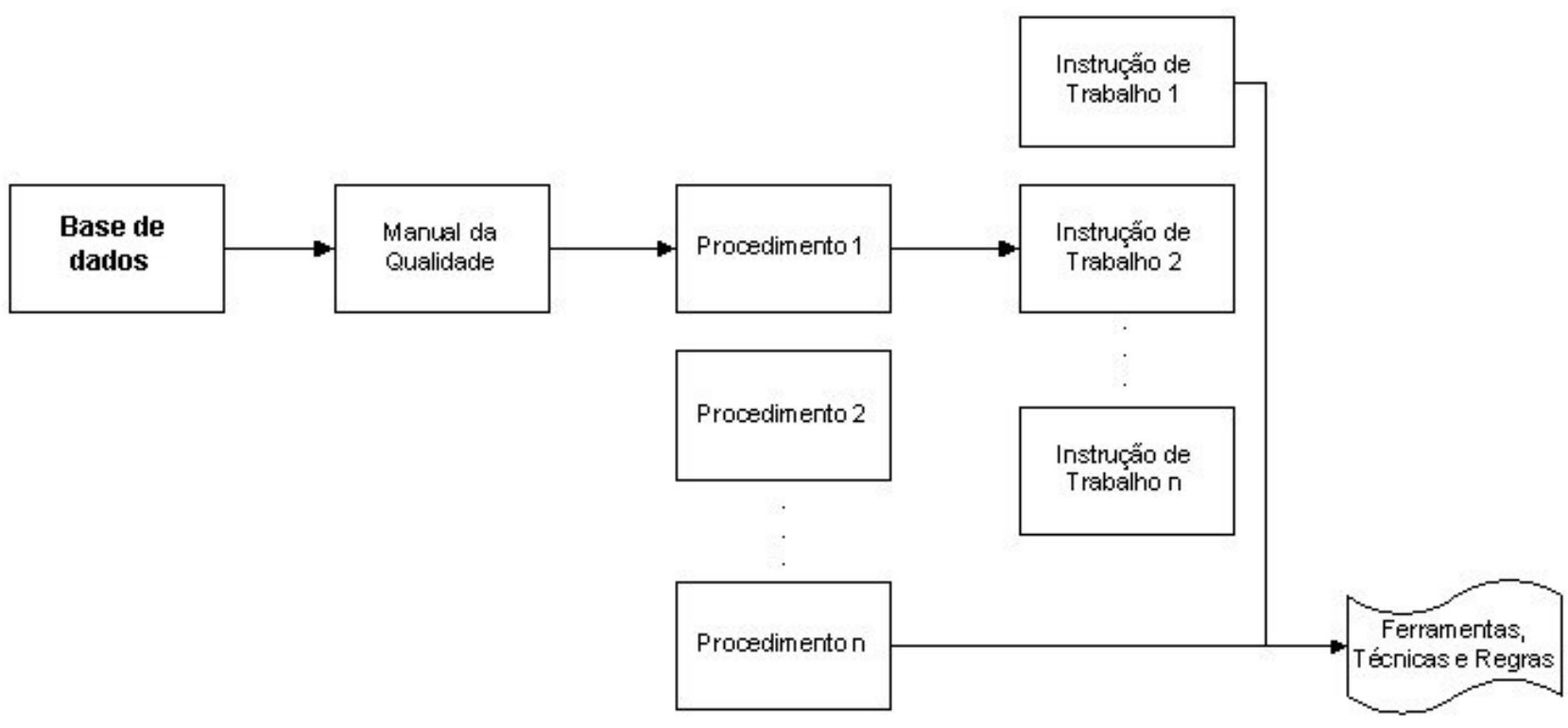

Figura 2: Estrutura dos processos da empresa analisada

Fonte: base de dados da empresa estudada (2016).

Os processos existentes na organização utilizam modelo PDCA (Plan, Do, Check, $A c t$ ) para um melhor entendimento do contexto e papel dos processos do sistema em 
uma abordagem de melhoria contínua. No Manual da Qualidade da organização (2016) cada item é definido da seguinte maneira: a) planejamento: atividades do sistema de gestão que são definidos pela elaboração e controle de documentos (procedimentos, instruções de trabalho e ferramentas/técnicas/regras); b) execução: realização dos processos - nesta rotina podem ser identificadas oportunidades de melhoria; c) verificação: que é realizado periodicamente no sistema através de análises críticas, pesquisas com os clientes e auditorias - outra fase que contribui para identificação de pontos de melhoria no sistema - e também são utilizados indicadores que fornecem números para uma análise mais precisa; d) correção e prevenção: os pontos de melhoria identificados são analisados pelo grupo de melhoria de processos da organização (EPG Engineering Process Group) e são definidas e realizadas ações corretivas e preventivas conforme necessário. Os processos preventivos abordam também a parte de aquisição e treinamento, bem como as atividades de manutenção do sistema informatizado.

Diante disto, temos o problema geral de pesquisa abordado nesta investigação, que é a falta de incorporação dos procedimentos e instruções de trabalho no dia a dia, o que acarreta na falta de participação para o continua melhoria dos processos e o desperdício da inteligência que pode ser gerado na organização. Pode-se perceber que há o esforço em gerar informação que contribua para a melhoria contínua da organização. Porém, essas ficam em repositórios pouco acessados, pela limitação da recuperação da informação enquanto enfoque informacional, o que dificulta a possibilidade da organização em reter e compartilhar o conhecimento. A falta de acesso a esses processos e a pouca contribuição em incrementá-los com experiências, feedback, revisões, os tornam obsoletos ou em desacordo com a realidade.

Compreende-se que a incorporações dos processos e possibilidades de melhorias, ao incrementar as experiências no processo, contribui para que as melhores decisões sejam tomadas com base no repositório de informação e conhecimento da organização.

Para estimular a incorporações dos processos e possibilitar melhorias na organização nas rotinas da organização, através das experiências, a atenção deve ser dada a recuperação da informação, no sentido de alcançar um efetivo processo de Memória Organizacional, dentro do escopo da Inteligência Organizacional.

\section{CONSIDERAÇÕES FINAIS (OU PARCIAIS)}

A literatura que sustenta este estudo é apresentada de forma a estabelecer as aproximações entre a recuperação da informação, memória organizacional e a inteligência organizacional, visando sustentar o diagnóstico de um problema em uma organização do ramo de tecnologia, quanto a melhoria do processo de tomada de decisão.

Como fonte do diagnóstico e detalhamento do problema foi utilizado o relatório gerado como resultado da avaliação externa denominado The Standard CMMI Appraisal Method for Process Improvement (SCAMPI) e as informações que constam na base de dados da organização.

Foi possível identificar que a organização possui uma política organizacional que orienta os colaboradores para a execução das atividades de acordo com os procedimentos, instruções de trabalho, ferramentas e regras, porém foi identificado deficiências no processo de recuperação da informação.

Desta forma, considera-se que um maior enfoque poderia ser dado a recuperação da informação, no sentido de alcançar efetivamente a Memória Organizacional, dentro do escopo da Inteligência Organizacional. Aspectos estes que contribuem para a otimização do 
processo de tomada de decisão, ao incrementar experiências que enriquecem o acervo de informação e conhecimento e permitir que estes sejam usados de forma de forma oportuna na organização.

Como recomendação a trabalhos futuros, outros enfoques informacionais poderiam ser explorados, desde geração à distribuição e uso da informação. Além disto, o estudo poderia ser realizado considerando como enfoque outros elementos da Inteligência Organizacional, tal como sentimento e comportamento adaptável.

\section{REFERÊNCIAS}

ABREU, J. C. A. A Democracia Digital e a Sociedade da Informação Sob Enfoque Gramsciano. In: Encontro de Administração da Informação, 2009. Recife. Anais... Recife: ENADI /ANPAD. 2009.

BASTOS, E. M. C. F. M. Políticas de Informação como fundamento para uma arquitetura de informação da Câmara dos Deputados: a análise do caso da política de indexação. 2013. Dissertação (Especialização do curso de. Arquitetura e Organização da Informação) - Escola de Ciência da Informação, UFMG, Minas Gerais, 2013.

CHOO, C. W. Aprendizado como inteligência organizacional. In: TARAPANOFF, Kira. Aprendizado organizacional. Curitiba: Ibpex, 2011.

COUTINHO, C. Sociedade da Informação, do conhecimento e da aprendizagem: desafios para a educação no século XXI. Revista de Educação, Vol. XVIII, no 1, p. 5-22, 2011. Disponível em: $<$ https://repositorium.sdum.uminho.pt $>$. Acesso em: 10 jan. 2017.

FLICK, U. Desenho da pesquisa qualitativa. Coleção Pesquisa qualitativa. Porto Alegre: Bookman Editora, 2009.

FREIRE, P. de S. et al. Memória organizacional e seu papel na gestão do conhecimento. Revista de Ciências da Administração, v. 14, n.33, p. 41-51, ago 2012. Disponível em: <https://periodicos.ufsc.br/index.php/adm/article/view/2175-8077.2012v14n33p41>. Acesso em: 10 jan. 2017.

GIL, A. C. Métodos e técnicas de pesquisa social. 6. ed. - São Paulo: Atlas, 2008.

GLYNN, M. A. Innovative genius: A framework for relating individual and organizational intelligences to innovation. Academy of Management Review, v.21, n.4, p. 1081-1111, 1996.

MANUAL da Qualidade. Base disponível na organização estudada. 2016.

MAXIMIANO, A. C. A. Introdução à Administração. São Paulo: Atlas, 2009.

MENEZES, E. M. Estruturação da memória organizacional de uma instituição em iminência de evasão de especialistas: um estudo de caso da Conab. 2006. 128 f. Dissertação (Mestrado) - Curso de Gestão do Conhecimento e da Tecnologia da Informação, Universidade Católica de Brasília, Brasília, 2006.

MOLINA, L. G; VALENTIN, M. L. P. Memória organizacional, memória corporativa e memória institucional: discussões conceituais e terminológicas. Revista EDICIC, v.1, n.1, p.262-276, Ene./Mar. 2011. Disponível em: <http://www.edicic.org/revista/>. Acesso em: 10 jan. 2017.

MÜLLER, R.; de CASTILHO JUNIOR, N. C. Inteligência organizacional como ferramenta de gestão: um referencial teórico integrado. Revista Expectativa, Toledo, v.11, n.11, p.83-102, jan/dez 2012. 
PRÉVE, A. D.; DE OLIVEIRA MORITZ, G.; PEREIRA, M. F. Organização, processos e tomada de decisão. Florianópolis: Departamento de Ciências da Administração/UFSC, 2010.

TARAPANOFF, K. Inteligência, informação e conhecimento em corporações. Brasília: Instituto Brasileiro de Informação em Ciência e Tecnologia (IBICT), 2006.

TEAM, SEI CMMI Production. CMMI for Development v1. 3. CMMI® Institute LLC, 2010.

TELLES, T. KARAWEJCZYK, C. K; BORGES, M. L. Memória organizacional: construção conceitual numa abordagem teórico-metodológica. VIII ENCONTRO DE ESTUDOS ORGANIZACIONAIS DA ANPAD. Anais... Gramado, 2014.

WALSH, J. P.; UNGSON, G. R. Organizational memory. Academy of Management Review, v.16, n.1, p. $57-91,1991$. 